ANNALES

POLONICI MATHEMATICI

$82.3(2003)$

\title{
Symplectic structures on the tangent bundles of symplectic and cosymplectic manifolds
}

\author{
by J. KureK (Lublin) and W. M. Mikulski (Kraków)
}

\begin{abstract}
We describe all natural symplectic structures on the tangent bundles of symplectic and cosymplectic manifolds.
\end{abstract}

0. Introduction. In [4], the authors classified all Hamiltonian type natural operators on the cotangent bundle $T^{*} M$. In [1], the author described all Hamiltonian type natural operators transforming a function $f$ on a symplectic manifold $(M, \omega)$ into a vector field $V(\omega, f)$ on $M$. The present paper also deals with natural operators on symplectic structures. We describe all natural symplectic structures on the tangent bundles of symplectic and cosymplectic manifolds. This problem arises in the context of not necessarily regular natural operators in the sense of I. Kolář, P. W. Michor and J. Slovak [6], which are defined on symplectic (resp. cosymplectic) structures. Since homotheties are not symplectomorphisms (resp. cosymplectomorphisms), it is difficult to apply the homogeneous function theorem and the problem of classification of the operators in question is more difficult than the one for natural operators defined on all 2 -forms (see [2], [3], [6], etc).

Symplectic structures are involved in the Hamilton equation of motion. For this reason the results of this paper are also interesting from the point of view of theoretical mechanics.

We start with the problem of how to construct canonically a symplectic manifold $(T M, \Lambda(\omega))$ for a given symplectic $2 m$-manifold $(M, \omega)$, where $\omega$ is a closed 2-form with $\omega^{m} \neq 0$ for any point in $M$. This problem arises in the context of not necessarily regular $\mathcal{M} f_{2 m}$-natural operators $\Lambda$, where $\mathcal{M} f_{2 m}$ is the category of $2 m$-dimensional manifolds and their local diffeomorphisms. We recall that a not necessarily regular $\mathcal{M} f_{2 m}$-natural operator in question

2000 Mathematics Subject Classification: Primary 58A20.

Key words and phrases: natural operators, symplectic structures, cosymplectic structures. 
is a family of functions

$$
\Lambda: \operatorname{SYMP}(M) \rightarrow \operatorname{SYMP}(T M)
$$

from $\operatorname{SYMP}(M)$, the set of all symplectic forms on $M$, into $\operatorname{SYMP}(T M)$ for any $2 m$-dimensional manifold $M$, satisfying the naturality condition

$$
\Lambda\left(\varphi^{*} \omega\right)=(T \varphi)^{*} \Lambda(\omega)
$$

for any symplectic form $\omega$ on a $2 m$-manifold $N$ and any local diffeomorphism $\varphi: M \rightarrow N$. The first main result of the present note is the following classification theorem.

THEOREM 1. Let $\Lambda$ be a not necessarily regular $\mathcal{M} f_{2 m}$-natural operator as above. Then there exist real numbers $\alpha$ and $\beta \neq 0$ such that

$$
\Lambda(\omega)=\alpha \pi^{*} \omega+\beta \widetilde{\omega}^{*} \Omega
$$

for any symplectic structure $\omega$ on $M$, where $\pi^{*} \omega$ is the vertical lifting of $\omega$ to the tangent bundle $T M, \pi: T M \rightarrow M$ is the tangent bundle projection, ()$^{*}$ is the pull-back, $\Omega$ is the canonical symplectic structure on $T^{*} M$ and $\widetilde{\omega}: T M \rightarrow T^{*} M$ is the standard isomorphism induced by $\omega, \widetilde{\omega}(v)=\omega_{x}(\cdot, v)$ for $v \in T_{x} M$ and $x \in M$.

Conversely, for any real numbers $\alpha$ and $\beta \neq 0$ the operator $\Lambda(\omega)$ defined by (1) is a symplectic structure on TM.

We recall that $\widetilde{\omega}^{*} \Omega$ coincides with the tangent lift $d_{T} \omega$ (W. Tulczyjew [8]), and (under the isomorphisms $T T M \rightarrow T^{*} T M$ induced by the symplectic form $\left.d_{T} \omega\right)$ with the tangent lift of the corresponding 2-vector field (J. Grabowski and P. Urbański [5]).

By the Darboux theorem every symplectic structure $\omega$ on a $2 m$-manifold $M$ is locally $\omega_{o}=\sum_{i=1}^{m} d x^{i} \wedge d x^{m+i}$ in some coordinate system $x^{j}$ on $M$. In the induced coordinate system $\left(x^{j}, y^{j}\right)$ on $T^{*} M$ we have $\Omega=\sum_{j=1}^{2 m} d x^{j} \wedge d y^{j}$. Hence $\Lambda(\omega)$ from (1) is a symplectic structure.

So, Theorem 1 is a consequence of the following fact.

THEOREM 2. Let $\Lambda$ be a not necessarily regular $\mathcal{M} f_{2 m}$-natural operator transforming a symplectic structure $\omega$ on a $2 m$-manifold $M$ into a 2-form $\Lambda(\omega)$ on $T M$. Then there exist real numbers $\alpha$ and $\beta$ such that

$$
\Lambda(\omega)=\alpha \pi^{*} \omega+\beta \widetilde{\omega}^{*} \Omega
$$

for any symplectic structure $\omega$ on $M$, where $\Omega$ and $\widetilde{\omega}$ are as in Theorem 1 .

The proof of Theorem 2 will occupy Section 1 of this note.

Using the isomorphism $\widetilde{\omega}: T M \rightarrow T^{*} M$ induced by $\omega$ we can obtain versions of Theorems 1 and 2 for $T^{*} M$ instead of $T M$.

Next, using Theorem 2, we study the problem of how to construct canonically a symplectic manifold $(T M, \Lambda(\omega, \theta))$ for a given cosymplectic $2 m+1$ manifold $(M, \omega, \theta)$, where $\omega$ is a closed 2-form and $\theta$ is a closed 1-form with 
$\omega^{m} \wedge \theta \neq 0$ for any point in $M$. This problem arises in the context of not necessarily regular $\mathcal{M} f_{2 m+1}$-natural operators $\Lambda$ (the definition is an obvious modification of the above one). Our second main result is the following classification theorem.

TheOREM 3. Let $\Lambda$ be a not necessarily regular $\mathcal{M} f_{2 m+1}$-natural operator as above. Then there exists a uniqely determined real number a and uniquely determined smooth maps $b, c: \mathbb{R} \rightarrow \mathbb{R}$ with $b(x) \neq 0$ and $b(x)+$ $c(x) \neq 0$ for all $x \in M$ such that

$$
\Lambda(\omega, \theta)=a \pi^{*} \omega+(b \circ \theta) \varphi_{\omega, \theta}^{*} \Omega+\left(b^{\prime} \circ \theta\right) \varphi_{\omega, \theta}^{*} \lambda \wedge d \theta+(c \circ \theta) \pi^{*} \theta \wedge d \theta
$$

for any cosymplectic structure $(\omega, \theta)$ on $M$, where $b^{\prime}: \mathbb{R} \rightarrow \mathbb{R}$ is the derivative of $b, \lambda$ is the standard Liouville 1-form on $T^{*} M, \Omega=-d \lambda$ is the canonical symplectic structure on $T^{*} M, \pi: T M \rightarrow M$ is the tangent bundle projection, $\varphi_{\omega, \theta}: T M \rightarrow T^{*} M$ is the standard isomorphism induced by $(\omega, \theta), \varphi_{\omega, \theta}(v)=\omega_{x}(\cdot, v)+\theta(v) \theta_{x}$ for $v \in T_{x} M$ and $x \in M,()^{*}$ is the pull-back and $d \theta$ is the differential of the fiber linear map $\theta: T M \rightarrow \mathbb{R}$ corresponding to the 1-form $\theta$.

Conversely, for any real number $a$ and smooth maps $b, c: \mathbb{R} \rightarrow \mathbb{R}$ with $b(x)+c(x) \neq 0$ and $b(x) \neq 0$ for all $x \in M$ the operator $\Lambda(\omega, \theta)$ defined as in (3) is a symplectic structure on TM.

By the Darboux theorem every cosymplectic structure $(\omega, \theta)$ on a $2 m+1$ manifold $M$ is locally $\omega_{o}=\sum_{i=1}^{m} d x^{i} \wedge d x^{m+i}$ and $\theta_{o}=d x^{2 m+1}$ in some coordinate system $x^{j}$ on $M$. In the induced coordinate system $\left(x^{j}, y^{j}\right)$ on $T^{*} M$ we have $\Omega=\sum_{j=1}^{2 m+1} d x^{j} \wedge d y^{j}, \pi^{*} \omega_{o}=\sum_{i=1}^{m} d x^{i} \wedge d x^{m+i}, \pi^{*} \theta_{o}=$ $d x^{2 m+1}$ and $d \theta_{o}=d y^{2 m+1}$. Hence $\Lambda(\omega, \theta)$ from (3) is a symplectic structure for any real number $a$ and any smooth maps $b, c: \mathbb{R} \rightarrow \mathbb{R}$ with $b(x)+c(x) \neq 0$ and $b(x) \neq 0$ for all $x \in M$.

In fact, in Section 2 we prove the following more general Theorem 4.

THEOREM 4. Let $\Lambda$ be a not necessarily regular $\mathcal{M} f_{2 m+1}$-natural operator transforming a cosymplectic structure $(\omega, \theta)$ on a $2 m+1$-manifold $M$ into a 2 -form $\Lambda(\omega, \theta)$ on TM. Then there exist uniquely determined smooth maps $\alpha, \beta, \gamma, \delta, \varepsilon: \mathbb{R} \rightarrow \mathbb{R}$ such that

$$
\begin{aligned}
\Lambda(\omega, \theta)= & (\alpha \circ \theta) \varphi_{\omega, \theta}^{*} \Omega+(\beta \circ \theta) \pi^{*} \omega+(\gamma \circ \theta) \varphi_{\omega, \theta}^{*} \lambda \wedge \pi^{*} \theta \\
& +(\delta \circ \theta) \varphi_{\omega, \theta}^{*} \lambda \wedge d \theta+(\varepsilon \circ \theta) \pi^{*} \theta \wedge d \theta
\end{aligned}
$$

for any cosymplectic structure $(\omega, \theta)$ on $M$, where $\lambda, \Omega=-d \lambda, \varphi_{\omega, \theta}, \pi$ and ( )* are as in Theorem 3 .

Theorem 3 can be fairly easily deduced from Theorem 4 . Just write (in the induced coordinates on $\left.T \mathbb{R}^{2 m+1}\right)$ that $\Lambda\left(\omega_{o}, \theta_{o}\right)$ from (4) is closed and next that $\left(\Lambda\left(\omega_{o}, \theta_{o}\right)\right)^{2 m+1} \neq 0$ everywhere, where $\left(\omega_{o}, \theta_{o}\right)$ is the above mentioned standard cosymplectic structure on $\mathbb{R}^{2 m+1}$. 
Using the isomorphism $\varphi_{\omega, \theta}: T M \rightarrow T^{*} M$ induced by $(\omega, \theta)$ we can obtain versions of Theorems 3 and 4 for $T^{*} M$ instead of $T M$.

Given a map $f: M \rightarrow \mathbb{R}$ on a manifold $M$ we have $f^{[0]}=f \circ \pi: T M \rightarrow \mathbb{R}$ and $f^{[1]}=d f: T M \rightarrow \mathbb{R}$.

The usual coordinates on $\mathbb{R}^{2 m}$ will be denoted by $x^{i}, x^{\bar{i}}$, where $i=$ $1, \ldots, m$ and $\bar{i}=m+i$. The standard symplectic structure on $\mathbb{R}^{2 m}$ is denoted by $\omega_{o}=\sum_{i=1}^{m} d x^{i} \wedge d x^{\bar{i}}$. The system $\left(x^{i}\right)^{[0]},\left(x^{\bar{i}}\right)^{[0]},\left(x^{i}\right)^{[1]},\left(x^{\bar{i}}\right)^{[1]}$ for $i=$ $1, \ldots, m$ is the induced coordinate system on $T \mathbb{R}^{2 m}$.

The usual coordinates on $\mathbb{R}^{2 m+1}$ will be denoted by $x^{i}, x^{\bar{i}}, x^{2 m+1}$, where $i=1, \ldots, m$ and $\bar{i}=m+i$. The standard cosymplectic structure on $\mathbb{R}^{2 m+1}$ is denoted by $\omega_{o}=\sum_{i=1}^{m} d x^{i} \wedge d x^{\bar{i}}$ and $\theta_{o}=d x^{2 m+1}$. The system $\left(x^{i}\right)^{[0]},\left(x^{\bar{i}}\right)^{[0]},\left(x^{i}\right)^{[1]},\left(x^{\bar{i}}\right)^{[1]},\left(x^{2 m+1}\right)^{[0]},\left(x^{2 m+1}\right)^{[1]}$ for $i=1, \ldots, m$ is the induced coordinate system on $T \mathbb{R}^{2 m+1}$.

All manifolds and maps are assumed to be of class $\mathcal{C}^{\infty}$.

1. The symplectic case. The purpose of this section is to prove Theorem 2 .

Using notations from Section 0, one can show that

$$
\begin{aligned}
\widetilde{\omega}_{o}^{*} \Omega & =\sum_{i=1}^{m} d\left(x^{i}\right)^{[0]} \wedge d\left(x^{\bar{i}}\right)^{[1]}-\sum_{i=1}^{m} d\left(x^{\bar{i}}\right)^{[0]} \wedge d\left(x^{i}\right)^{[1]}, \\
\pi^{*} \omega_{o} & =\sum_{i=1}^{m} d\left(x^{i}\right)^{[0]} \wedge d\left(x^{\bar{i}}\right)^{[0]} .
\end{aligned}
$$

Let $\Lambda$ be as in Theorem 2 .

Lemma 1. If $\Lambda\left(\omega_{o}\right)=0$, then $\Lambda=0$.

Proof. We have to show that $\Lambda(\omega)=0$ for any symplectic structure $\omega$ on a $2 m$-manifold. By the Darboux theorem and naturality we can assume $\omega=\omega_{o}$.

In view of Lemma $1, \Lambda$ is uniquely determined by $\Lambda\left(\omega_{o}\right)$. So, it is sufficient to study $\Lambda\left(\omega_{o}\right)$. By the invariance of $\Lambda\left(\omega_{o}\right)$ with respect to translations $\mathbb{R}^{2 m} \rightarrow \mathbb{R}^{2 m}$ we can write

$$
\begin{aligned}
\Lambda\left(\omega_{0}\right)= & \sum_{(k, \lambda)<(l, \mu)} f_{(k, \lambda),(l, \mu)}\left(\left(x^{i}\right)^{[1]},\left(x^{\bar{i}}\right)^{[1]}\right) d\left(x^{k}\right)^{[\lambda]} \wedge d\left(x^{l}\right)^{[\mu]} \\
& +\sum_{(k, \lambda)<(l, \mu)} g_{(k, \lambda),(l, \mu)}\left(\left(x^{i}\right)^{[1]},\left(x^{\bar{i}}\right)^{[1]}\right) d\left(x^{\bar{k}}\right)^{[\lambda]} \wedge d\left(x^{\bar{l}}\right)^{[\mu]} \\
& +\sum_{k, l=1}^{m} \sum_{\lambda, \mu=0,1} h_{(k, \lambda),(l, \mu)}\left(\left(x^{i}\right)^{[1]},\left(x^{\bar{i}}\right)^{[1]}\right) d\left(x^{k}\right)^{[\lambda]} \wedge d\left(x^{\bar{l}}\right)^{[\mu]}
\end{aligned}
$$


for some smooth maps $f_{(k, \lambda),(l, \mu)}, g_{(k, \lambda),(l, \mu)}, h_{(k, \lambda),(l, \mu)}: \mathbb{R}^{2 m} \rightarrow \mathbb{R}$, where the $\operatorname{sum} \sum_{(k, \lambda)<(l, \mu)}$ is over all $(k, \lambda),(l, \mu) \in\{1, \ldots, m\} \times\{0,1\}$ with $(k, \lambda)<(l, \mu)$ with respect to the lexicographic ordering on $\{1, \ldots, m\} \times$ $\{0,1\}$ (i.e. $k<l$ or $(k=l$ and $\lambda<\mu))$ and $\bar{k}=m+k, \bar{l}=m+l, \bar{i}=m+i$.

We now study the maps $f_{(k, \lambda),(l, \mu)}, g_{(k, \lambda),(l, \mu)}: \mathbb{R}^{2 m} \rightarrow \mathbb{R}$.

Lemma 2. For any $(k, \lambda),(l, \mu) \in\{1, \ldots, m\} \times\{0,1\}$ with $(k, \lambda)<(l, \mu)$ there exists a real number $a_{(k, \lambda),(l, \mu)}$ such that

$$
f_{(k, \lambda),(l, \mu)}\left(\left(x^{i}\right)^{[1]},\left(x^{\bar{i}}\right)^{[1]}\right)=a_{(k, \lambda),(l, \mu)}\left(x^{\bar{k}}\right)^{[1]}\left(x^{\bar{l}}\right)^{[1]} .
$$

For any $(k, \lambda),(l, \mu) \in\{1, \ldots, m\} \times\{0,1\}$ with $(k, \lambda)<(l, \mu)$ there exists a real number $b_{(k, \lambda),(l, \mu)}$ such that

$$
g_{(k, \lambda),(l, \mu)}\left(\left(x^{i}\right)^{[1]},\left(x^{\bar{i}}\right)^{[1]}\right)=b_{(k, \lambda),(l, \mu)}\left(x^{k}\right)^{[1]}\left(x^{l}\right)^{[1]} .
$$

Proof. Using the invariance of $\Lambda\left(\omega_{o}\right)$ with respect to the $\omega_{o}$-automorphisms $\bar{x}^{i}=x^{i}-\tau_{i} x^{\bar{i}}$ and $\bar{x}^{\bar{i}}=x^{\bar{i}}$ for $i=1, \ldots, m$ and arbitrary real numbers $\tau_{i}$ from (7) we get the conditions

$$
f_{(k, \lambda),(l, \mu)}\left(\left(x^{i}\right)^{[1]}+\tau_{i}\left(x^{\bar{i}}\right)^{[1]},\left(x^{\bar{i}}\right)^{[1]}\right)=f_{(k, \lambda),(l, \mu)}\left(\left(x^{i}\right)^{[1]},\left(x^{\bar{i}}\right)^{[1]}\right)
$$

Thus the maps $f_{(k, \lambda),(l, \mu)}$ are independent of the first $m$ coordinates.

By the same argument for the $\omega_{o}$-automorphisms $\bar{x}^{\bar{i}}=x^{\bar{i}}-\tau_{i} x^{i}$ and $\bar{x}^{i}=x^{i}$ for $i=1, \ldots, m$ we deduce that the maps $g_{(k, \lambda),(l, \mu)}$ are independent of the last $m$ coordinates.

Then by the invariance of $\Lambda\left(\omega_{o}\right)$ with respect to the $\omega_{o}$-automorphisms $\bar{x}^{i}=\tau_{i} x^{i}$ and $\bar{x}^{\bar{i}}=\left(1 / \tau_{i}\right) x^{\bar{i}}$ for $i=1, \ldots, m$ and arbitrary positive real numbers $\tau_{i}$ we get the homogeneity conditions

$$
\begin{gathered}
f_{(k, \lambda),(l, \mu)}\left(\tau_{i}\left(x^{\bar{i}}\right)^{[1]}\right)=\tau_{k} \tau_{l} f_{(k, \lambda),(l, \mu)}\left(\left(x^{\bar{i}}\right)^{[1]}\right), \\
g_{(k, \lambda),(l, \mu)}\left(\frac{1}{\tau_{i}}\left(x^{i}\right)^{[1]}\right)=\frac{1}{\tau_{k}} \frac{1}{\tau_{l}} g_{(k, \lambda),(l, \mu)}\left(\left(x^{i}\right)^{[1]}\right) .
\end{gathered}
$$

Now an application of the homogeneous function theorem finishes the proof.

Next, we study the maps $h_{(k, \lambda),(l, \mu)}: \mathbb{R}^{2 m} \rightarrow \mathbb{R}$.

Lemma 3. For any $(k, \lambda),(l, \mu) \in\{1, \ldots, m\} \times\{0,1\}$ with $k \neq l$ there exists a real number $c_{(k, \lambda),(l, \mu)}$ such that

$$
h_{(k, \lambda),(l, \mu)}\left(\left(x^{i}\right)^{[1]},\left(x^{\bar{i}}\right)^{[1]}\right)=c_{(k, \lambda),(l, \mu)}\left(x^{\bar{k}}\right)^{[1]}\left(x^{l}\right)^{[1]} .
$$

Proof. Let $k_{o}, l_{o} \in\{1, \ldots, m\}$ with $k_{o}<l_{o}$. Using Lemma 2 and the invariance of $\Lambda\left(\omega_{o}\right)$ with respect to the $\omega_{o}$-automorphisms $\bar{x}^{l_{o}}=x^{\bar{l}_{o}}, \bar{x}^{\bar{l}_{o}}=$ 
$-x^{l_{o}}$ and $\bar{x}^{i}=x^{i}$ and $\bar{x}^{\bar{i}}=x^{\bar{i}}$ for $i=1, \ldots, m$ with $i \neq l_{o}$ we get

$$
\begin{aligned}
& \sum_{\lambda, \mu=0,1} h_{\left(k_{o}, \lambda\right),\left(l_{o}, \mu\right)}\left(\left(x^{1}\right)^{[1]}, \ldots,\left(x^{\bar{l}_{o}}\right)^{[1]}, \ldots,\left(x^{m}\right)^{[1]},\right. \\
& \left.\left.\left(x^{\overline{1}}\right)^{[1]}\right), \ldots,-\left(x^{l_{o}}\right)^{[1]}, \ldots,\left(x^{\bar{m}}\right)^{[1]}\right) d\left(x^{k_{o}}\right)^{[\lambda]} \wedge d\left(x^{l_{o}}\right)^{[\mu]} \\
& =-\sum_{\lambda, \mu=0,1} a_{\left(k_{o}, \lambda\right),\left(l_{o}, \mu\right)}\left(x^{\bar{k}_{o}}\right)^{[1]}\left(x^{\bar{l}_{o}}\right)^{[1]} d\left(x^{k_{o}}\right)^{[\lambda]} \wedge d\left(x^{l_{o}}\right)^{[\mu]} .
\end{aligned}
$$

This ends the proof in the case $k<l$. The case $k>l$ is quite similar.

Lemma 4. For the constants of Lemmas 2 and 3, we have

$$
a_{(k, \lambda),(l, \mu)}=0, \quad b_{(k, \lambda),(l, \mu)}=0
$$

for any $(k, \lambda),(l, \mu) \in\{1, \ldots, m\} \times\{0,1\}$ with $k<l$, and

$$
c_{(k, \lambda),(l, \mu)}=0
$$

for any $(k, \lambda),(l, \mu) \in\{1, \ldots, m\} \times\{0,1\}$ with $k \neq l$.

Proof. Let $k_{o}, l_{o} \in\{1, \ldots, m\}$ with $k_{o}<l_{o}$. Using the invariance of $\Lambda\left(\omega_{o}\right)$ with respect to the $\omega_{o}$-automorphisms $\bar{x}^{i}=x^{i}-\tau_{i} x^{\bar{i}}$ and $\bar{x}^{\bar{i}}=x^{\bar{i}}$ for $i=1, \ldots, m$ and arbitrary real numbers $\tau_{i}$ we get an equality with both sides being polynomials in $\tau_{k_{o}}$ and $\tau_{l_{o}}$. Considering the coefficients of $\tau_{k_{o}}$ and $\tau_{l_{o}}$ we get (12). Considering the coefficients of $\left(\tau_{k_{o}}\right)^{2}\left(\tau_{l_{o}}\right)^{2}$ we get the second equality of (11). Then considering the coefficients of $\tau_{k_{o}} \tau_{l_{o}}$ we get the first equality of (11).

By (7) and Lemmas 2-4 we can write

$$
\begin{aligned}
\Lambda\left(\omega_{o}\right)= & \sum_{k=1}^{m} A_{k}\left(\left(x^{\bar{k}}\right)^{[1]}\right)^{2} d\left(x^{k}\right)^{[0]} \wedge d\left(x^{k}\right)^{[1]} \\
& +\sum_{k=1}^{m} B_{k}\left(\left(x^{k}\right)^{[1]}\right)^{2} d\left(x^{\bar{k}}\right)^{[0]} \wedge d\left(x^{\bar{k}}\right)^{[1]} \\
& +\sum_{k=1}^{m} \sum_{\lambda, \mu=0,1} C_{k, \lambda, \mu}\left(\left(x^{i}\right)^{[1]},\left(x^{\bar{i}}\right)^{[1]}\right) d\left(x^{k}\right)^{[\lambda]} \wedge d\left(x^{\bar{k}}\right)^{[\mu]}
\end{aligned}
$$

for some real numbers $A_{k}, B_{k}$ and some maps $C_{k, \lambda, \mu}: \mathbb{R}^{2 m} \rightarrow \mathbb{R}$.

Consider now a local germ ${ }_{0}\left(\omega_{o}\right)$-preserving diffeomorphism

$$
\varphi=\left(x^{i}-\frac{1}{2}\left(x^{i}\right)^{2}, \frac{x^{\bar{i}}}{1-x^{i}}\right): U \rightarrow \mathbb{R}^{2 m},
$$

where $U$ is a neighbourhood of $0 \in \mathbb{R}^{2 m}$.

Lemma 5. For $i=1, \ldots, m$ we have

$$
\left(x^{i}\right)^{[1]} \circ T \varphi=\left(x^{i}\right)^{[1]}, \quad\left(x^{\bar{i}}\right)^{[1]} \circ T \varphi=\left(x^{\bar{i}}\right)^{[1]},
$$




$$
\begin{gathered}
d\left(x^{i}\right)^{[0]} \circ T T \varphi=d\left(x^{i}\right)^{[0]}, \quad d\left(x^{\bar{i}}\right)^{[0]} \circ T T \varphi=d\left(x^{\bar{i}}\right)^{[0]}, \\
d\left(x^{i}\right)^{[1]} \circ T T \varphi=d\left(x^{i}\right)^{[1]}-\left(x^{i}\right)^{[1]} d\left(x^{i}\right)^{[0]}, \\
d\left(x^{\bar{i}}\right)^{[1]} \circ T T \varphi=d\left(x^{\bar{i}}\right)^{[1]}+\left(x^{\bar{i}}\right)^{[1]} d\left(x^{i}\right)^{[0]}+\left(x^{i}\right)^{[1]} d\left(x^{\bar{i}}\right)^{[0]}
\end{gathered}
$$

over $0 \in \mathbb{R}^{2 m}$.

Proof. It is clear that

$$
\begin{aligned}
& \left(x^{i}\right)^{[0]} \circ T \varphi=\left(x^{i}\right)^{[0]}-\frac{1}{2}\left(\left(x^{i}\right)^{[0]}\right)^{2}, \\
& \left(x^{\bar{i}}\right)^{[0]} \circ T \varphi=\frac{\left(x^{\bar{i}}\right)^{[0]}}{1-\left(x^{i}\right)^{[0]}} \\
& \left(x^{i}\right)^{[1]} \circ T \varphi=\left(1-\left(x^{i}\right)^{[0]}\right)\left(x^{i}\right)^{[1]}, \\
& \left(x^{\bar{i}}\right)^{[1]} \circ T \varphi=\frac{1}{1-\left(x^{i}\right)^{[0]}}\left(x^{\bar{i}}\right)^{[1]}+\frac{\left(x^{\bar{i}}\right)^{[0]}}{\left(1-\left(x^{i}\right)^{[0]}\right)^{2}}\left(x^{i}\right)^{[1]}
\end{aligned}
$$

over $U$. Differentiating both sides of each equality and using the fact that $\left(x^{i}\right)^{[0]}=0$ and $\left(x^{\bar{i}}\right)^{[0]}=0$ over $0 \in \mathbb{R}^{2 m}$ we complete the proof.

Lemma 6. For the coefficients in (13), we have

$$
\begin{gathered}
C_{k, 1,1}=0, \quad A_{k}=B_{k}, \\
-B_{k}\left(\left(x^{k}\right)^{[1]}\right)^{2}\left(x^{\bar{k}}\right)^{[1]}-C_{k, 1,0}\left(x^{k}\right)^{[1]}+C_{k, 0,1}\left(x^{k}\right)^{[1]}=0, \\
C_{k, 1,0}+C_{k, 0,1}=2 D_{k}, \quad C_{k, 0,0}=E_{k}
\end{gathered}
$$

for any $k=1, \ldots, m$, where $D_{k}$ and $E_{k}$ are real numbers. From (19) and (20) we get

$$
C_{k, 0,1}=\frac{1}{2} B_{k}\left(x^{k}\right)^{[1]}\left(x^{\bar{k}}\right)^{[1]}+D_{k}, \quad C_{k, 1,0}=-\frac{1}{2} B_{k}\left(x^{k}\right)^{[1]}\left(x^{\bar{k}}\right)^{[1]}+D_{k} .
$$

Proof. Let $\varphi$ be as in Lemma 5. Clearly $\varphi$ preserves the germ of $\omega_{o}$ at 0 . Then using the invariance of $\Lambda\left(\omega_{o}\right)$ over $0 \in \mathbb{R}^{2 m}$ with respect to $\varphi^{-1}$ and using the transformation rules (Lemma 5) from (13) we get a certain equality.

Considering the coefficients of $d\left(x^{k}\right)^{[1]} \wedge d\left(x^{\bar{k}}\right)^{[0]}$ of both sides of that equality we get $C_{k, 1,1}=0$.

Then considering the coefficients of $\left.d\left(x^{k}\right)^{[0]} \wedge d(x)^{\bar{k}}\right)^{[0]}$ we get (19).

Using the invariance of $\Lambda\left(\omega_{0}\right)$ with respect to the $\omega_{o}$-automorphism $\bar{x}^{i}=$ $-x^{\bar{i}}$ and $\bar{x}^{\bar{i}}=x^{i}$ from (13) we deduce the second formula in (18).

Using the invariance of $\Lambda\left(\omega_{0}\right)$ with respect to the $\omega_{0}$-automorphisms $\bar{x}^{i}=x^{i}-\tau_{i} x^{\bar{i}}$ and $\bar{x}^{\bar{i}}=x^{\bar{i}}$ for $i=1, \ldots, m$ and arbitrary real numbers $\tau_{i}$ we get 


$$
\begin{aligned}
-\tau_{k} A_{k}\left(\left(x^{\bar{k}}\right)^{[1]}\right)^{2}+C_{k, 1,0}\left(\left(x^{i}\right)^{[1]}+\tau_{i}\left(x^{\bar{i}}\right)^{[1]},\left(x^{\bar{i}}\right)^{[1]}\right) & =C_{k, 1,0}\left(\left(x^{i}\right)^{[1]},\left(x^{\bar{i}}\right)^{[1]}\right), \\
\tau_{k} A_{k}\left(\left(x^{\bar{k}}\right)^{[1]}\right)^{2}+C_{k, 0,1}\left(\left(x^{i}\right)^{[1]}+\tau_{i}\left(x^{\bar{i}}\right)^{[1]},\left(x^{\bar{i}}\right)^{[1]}\right) & =C_{k, 0,1}\left(\left(x^{i}\right)^{[1]},\left(x^{\bar{i}}\right)^{[1]}\right) .
\end{aligned}
$$

Summing the equalities we find that $C_{k, 1,0}+C_{k, 0,1}$ is independent of the first $m$ coordinates. Similarly one can prove that $C_{k, 1,0}+C_{k, 0,1}$ is independent of the last $m$ coordinates.

Using the invariance of $\Lambda\left(\omega_{o}\right)$ with respect to the $\omega_{o}$-automorphisms $\bar{x}^{i}=x^{i}-\tau_{i} x^{\bar{i}}$ and $\bar{x}^{\bar{i}}=x^{\bar{i}}$ for $i=1, \ldots, m$ and arbitrary real numbers $\tau_{i}$ we get

$$
C_{k, 0,0}\left(\left(x^{i}\right)^{[1]}+\tau_{i}\left(x^{\bar{i}}\right)^{[1]},\left(x^{\bar{i}}\right)^{[1]}\right)=C_{k, 0,0}\left(\left(x^{i}\right)^{[1]},\left(x^{\bar{i}}\right)^{[1]}\right) .
$$

So, $C_{k, 0,0}$ is independent of the first $m$ coordinates. Similarly, $C_{k, 0,0}$ is independent of the last $m$ coordinates.

By (13) and Lemma 6 we can write

$$
\begin{aligned}
\Lambda\left(\omega_{o}\right)= & \sum_{k=1}^{m} B_{k}\left(\left(x^{\bar{k}}\right)^{[1]}\right)^{2} d\left(x^{k}\right)^{[0]} \wedge d\left(x^{k}\right)^{[1]} \\
& +\sum_{k=1}^{m} B_{k}\left(\left(x^{k}\right)^{[1]}\right)^{2} d\left(x^{\bar{k}}\right)^{[0]} \wedge d\left(x^{\bar{k}}\right)^{[1]} \\
& +\frac{1}{2} \sum_{k=1}^{m} B_{k}\left(x^{k}\right)^{[1]}\left(x^{\bar{k}}\right)^{[1]} d\left(x^{k}\right)^{[0]} \wedge d\left(x^{\bar{k}}\right)^{[1]} \\
& -\frac{1}{2} \sum_{k=1}^{m} B_{k}\left(x^{k}\right)^{[1]}\left(x^{\bar{k}}\right)^{[1]} d\left(x^{k}\right)^{[1]} \wedge d\left(x^{\bar{k}}\right)^{[0]} \\
& +\sum_{k=1}^{m} D_{k} d\left(x^{k}\right)^{[0]} \wedge d\left(x^{\bar{k}}\right)^{[1]}-\sum_{k=1}^{m} D_{k} d\left(x^{\bar{k}}\right)^{[0]} \wedge d\left(x^{k}\right)^{[1]} \\
& +\sum_{k=1}^{m} E_{k} d\left(x^{k}\right)^{[0]} \wedge d\left(x^{\bar{k}}\right)^{[0]}
\end{aligned}
$$

for some real numbers $B_{k}, E_{k}, D_{k}$.

Using the invariance of $\Lambda\left(\omega_{o}\right)$ with respect to permutations of the first $m$ coordinates together with the corresponding permutations of the last $m$ coordinates we deduce that $B_{k}=B$ and $D_{k}=D$ and $E_{k}=E$ for any $k=1, \ldots, m$. So, replacing $\Lambda(\omega)$ by $\Lambda(\omega)-E \pi^{*} \omega-D \widetilde{\omega}^{*} \Omega$ we have

$$
\begin{aligned}
\Lambda\left(\omega_{o}\right)= & B \sum_{k=1}^{m}\left(\left(x^{\bar{k}}\right)^{[1]}\right)^{2} d\left(x^{k}\right)^{[0]} \wedge d\left(x^{k}\right)^{[1]} \\
& +B \sum_{k=1}^{m}\left(\left(x^{k}\right)^{[1]}\right)^{2} d\left(x^{\bar{k}}\right)^{[0]} \wedge d\left(x^{\bar{k}}\right)^{[1]}
\end{aligned}
$$




$$
\begin{aligned}
& +\frac{1}{2} B \sum_{k=1}^{m}\left(x^{k}\right)^{[1]}\left(x^{\bar{k}}\right)^{[1]} d\left(x^{k}\right)^{[0]} \wedge d\left(x^{\bar{k}}\right)^{[1]} \\
& -\frac{1}{2} B \sum_{k=1}^{m}\left(x^{k}\right)^{[1]}\left(x^{\bar{k}}\right)^{[1]} d\left(x^{k}\right)^{[1]} \wedge d\left(x^{\bar{k}}\right)^{[0]}
\end{aligned}
$$

for some real number $B$.

Consider now the $\omega_{0}$-diffeomorphism

$$
\psi=\left(x^{i}-\left(x^{\bar{i}}\right)^{2}, x^{\bar{i}}\right) .
$$

LEMma 7. For $i=1, \ldots, m$ we have

$$
\begin{gathered}
\left(x^{i}\right)^{[1]} \circ T \psi=\left(x^{i}\right)^{[1]}, \quad\left(x^{\bar{i}}\right)^{[1]} \circ T \psi=\left(x^{\bar{i}}\right)^{[1]}, \\
d\left(x^{i}\right)^{[0]} \circ T T \psi=d\left(x^{i}\right)^{[0]}, \quad d\left(x^{\bar{i}}\right)^{[0]} \circ T T \psi=d\left(x^{\bar{i}}\right)^{[0]}, \\
d\left(x^{i}\right)^{[1]} \circ T T \psi=d\left(x^{i}\right)^{[1]}-2\left(x^{\bar{i}}\right)^{[1]} d\left(x^{\bar{i}}\right)^{[0]}, \\
d\left(x^{\bar{i}}\right)^{[1]} \circ T T \psi=d\left(x^{\bar{i}}\right)^{[1]}
\end{gathered}
$$

over $0 \in \mathbb{R}^{2 m}$.

Proof. The proof is quite similar to the one of Lemma 5.

Proof of Theorem 2. By Lemma 1 it is sufficient to prove that $B=0$, where $B$ is as in (23).

Let $\psi$ be as in Lemma 7 . Using the invariance of $\Lambda\left(\omega_{o}\right)$ with respect to $\psi^{-1}$ from (23) and the transformation rules from Lemma 7 we get $B=0$.

2. The cosymplectic case. The purpose of this section is to prove Theorem 4.

Using notations from Section 0, one can prove that

$$
\begin{aligned}
\varphi_{\omega_{o}, \theta_{o}}^{*} \Omega= & \sum_{i=1}^{m} d\left(x^{i}\right)^{[0]} \wedge d\left(x^{\bar{i}}\right)^{[1]}-\sum_{i=1}^{m} d\left(x^{\bar{i}}\right)^{[0]} \wedge d\left(x^{i}\right)^{[1]} \\
& +d\left(x^{2 m+1}\right)^{[0]} \wedge d\left(x^{2 m+1}\right)^{[1]} \\
\varphi_{\omega_{o}, \theta_{o}}^{*} \lambda \wedge \pi^{*} \theta_{o}= & -\sum_{i=1}^{m}\left(x^{i}\right)^{[1]} d\left(x^{\bar{i}}\right)^{[0]} \wedge d\left(x^{2 m+1}\right)^{[0]} \\
& +\sum_{i=1}^{m}\left(x^{\bar{i}}\right)^{[1]} d\left(x^{i}\right)^{[0]} \wedge d\left(x^{2 m+1}\right)^{[0]} \\
\varphi_{\omega_{o}, \theta_{o}}^{*} \lambda \wedge d \theta_{o}= & -\sum_{i=1}^{m}\left(x^{i}\right)^{[1]} d\left(x^{\bar{i}}\right)^{[0]} \wedge d\left(x^{2 m+1}\right)^{[1]} \\
& +\sum_{i=1}^{m}\left(x^{\bar{i}}\right)^{[1]} d\left(x^{i}\right)^{[0]} \wedge d\left(x^{2 m+1}\right)^{[1]}
\end{aligned}
$$




$$
\pi^{*} \theta_{o} \wedge d \theta_{o}=d\left(x^{2 m+1}\right)^{[0]} \wedge d\left(x^{2 m+1}\right)^{[1]} .
$$

Let $\Lambda$ be as in Theorem 4 .

LEMMA 8. If $\Lambda\left(\omega_{o}, \theta_{o}\right)=0$, then $\Lambda=0$.

Proof. We have to show that $\Lambda(\omega, \theta)=0$ for any cosymplectic structure $(\omega, \theta)$ on a $2 m+1$-manifold. By the Darboux theorem and the naturality we can assume $\omega=\omega_{o}$ and $\theta=\theta_{o}$.

So, it is sufficient to study $\Lambda\left(\omega_{o}, \theta_{o}\right)$. Given $c \in \mathbb{R}$, let

$$
i_{c}: T \mathbb{R}^{2 m}=\mathbb{R}^{2 m} \times \mathbb{R}^{2 m} \rightarrow \mathbb{R}^{2 m} \times\{0\} \times \mathbb{R}^{2 m} \times\{c\} \subset T \mathbb{R}^{2 m+1}
$$

be the inclusion.

LEMMA 9. We have

$$
\begin{aligned}
\left(i_{c}\right)^{*} \Lambda\left(\omega_{o}, \theta_{o}\right)= & a(c)\left(\sum_{i=1}^{m} d\left(x^{i}\right)^{[0]} \wedge d\left(x^{\bar{i}}\right)^{[1]}-\sum_{i=1}^{m} d\left(x^{\bar{i}}\right)^{[0]} \wedge d\left(x^{i}\right)^{[1]}\right) \\
& +b(c) \sum_{i=1}^{m} d\left(x^{i}\right)^{[0]} \wedge d\left(x^{\bar{i}}\right)^{[0]},
\end{aligned}
$$

for some smooth maps $a, b: \mathbb{R} \rightarrow \mathbb{R}$.

Proof. The 2-form $\left(i_{c}\right)^{*} \Lambda\left(\omega_{o}, \theta_{o}\right)$ on $T \mathbb{R}^{2 m}$ is clearly invariant with respect to all symplectomorphisms $\mathbb{R}^{2 m} \rightarrow \mathbb{R}^{2 m}$, where on $\mathbb{R}^{2 m}$ we have the standard symplectic form. An appeal to Theorem 2 completes the proof.

By the invariance of $\Lambda\left(\omega_{o}, \theta_{o}\right)$ with respect to translations $\mathbb{R}^{2 m+1} \rightarrow$ $\mathbb{R}^{2 m+1}$ and by Lemma 9 we can write

$$
\begin{aligned}
& \Lambda\left(\omega_{o}, \theta_{o}\right) \\
= & a\left(\left(x^{2 m+1}\right)^{[1]}\right)\left(\sum_{i=1}^{m} d\left(x^{i}\right)^{[0]} \wedge d\left(x^{\bar{i}}\right)^{[1]}-\sum_{i=1}^{m} d\left(x^{\bar{i}}\right)^{[0]} \wedge d\left(x^{i}\right)^{[1]}\right) \\
& +b\left(\left(x^{2 m+1}\right)^{[1]}\right) \sum_{i=1}^{m} d\left(x^{i}\right)^{[0]} \wedge d\left(x^{\bar{i}}\right)^{[0]} \\
& +\sum_{k=1}^{m} \sum_{\lambda, \mu=0,1} F_{k, \mu, \nu}\left(\left(x^{i}\right)^{[1]},\left(x^{\bar{i}}\right)^{[1]},\left(x^{2 m+1}\right)^{[1]}\right) d\left(x^{k}\right)^{[\mu]} \wedge d\left(x^{2 m+1}\right)^{[\nu]} \\
& +\sum_{k=1}^{m} \sum_{\lambda, \mu=0,1} G_{k, \mu, \nu}\left(\left(x^{i}\right)^{[1]},\left(x^{\bar{i}}\right)^{[1]},\left(x^{2 m+1}\right)^{[1]}\right) d\left(x^{\bar{k}}\right)^{[\mu]} \wedge d\left(x^{2 m+1}\right)^{[\nu]} \\
& +H\left(\left(x^{i}\right)^{[1]},\left(x^{\bar{i}}\right)^{[1]},\left(x^{2 m+1}\right)^{[1]}\right) d\left(x^{2 m+1}\right)^{[0]} \wedge d\left(x^{2 m+1}\right)^{[1]}
\end{aligned}
$$

for some smooth maps $a, b: \mathbb{R} \rightarrow \mathbb{R}$ and $F_{k, \mu, \nu}, G_{k, \mu, \nu}, H: \mathbb{R}^{2 m+1} \rightarrow \mathbb{R}$, where $\bar{k}=k+m, \bar{i}=m+i$. 
Lemma 10. For any $k \in\{1, \ldots, m\}$ and $\mu, \nu \in\{0,1\}$ there are smooth maps $a_{k, \mu, \nu}$ and $b_{k, \mu, \nu}$ such that

$$
\begin{aligned}
& F_{k, \mu, \nu}\left(\left(x^{i}\right)^{[1]},\left(x^{\bar{i}}\right)^{[1]},\left(x^{2 m+1}\right)^{[1]}\right)=a_{k, \mu, \nu}\left(\left(x^{2 m+1}\right)^{[1]}\right)\left(x^{\bar{k}}\right)^{[1]} \\
& G_{k, \mu, \nu}\left(\left(x^{i}\right)^{[1]},\left(x^{\bar{i}}\right)^{[1]},\left(x^{2 m+1}\right)^{[1]}\right)=b_{k, \mu, \nu}\left(\left(x^{2 m+1}\right)^{[1]}\right)\left(x^{k}\right)^{[1]}
\end{aligned}
$$

We can write

$$
H\left(\left(x^{i}\right)^{[1]},\left(x^{\bar{i}}\right)^{[1]},\left(x^{2 m+1}\right)^{[1]}\right)=c\left(\left(x^{2 m+1}\right)^{[1]}\right)
$$

for some smooth map $c: \mathbb{R} \rightarrow \mathbb{R}$.

Proof. Using the invariance of $\Lambda\left(\omega_{o}, \theta_{o}\right)$ with respect to the $\left(\omega_{o}, \theta_{o}\right)$ automorphisms $\bar{x}^{i}=x^{i}-\tau_{i} x^{\bar{i}}, \bar{x}^{\bar{i}}=x^{\bar{i}}$ and $\bar{x}^{2 m+1}=x^{2 m+1}$ for $i=1, \ldots, m$ and arbitrary real numbers $\tau_{i}$, from (33) we get

$$
\begin{gathered}
F_{k, \mu, \nu}\left(\left(x^{i}\right)^{[1]}+\tau_{i}\left(x^{\bar{i}}\right)^{[1]},\left(x^{\bar{i}}\right)^{[1]},\left(x^{2 m+1}\right)^{[1]}\right) \\
=F_{k, \mu, \nu}\left(\left(x^{i}\right)^{[1]},\left(x^{\bar{i}}\right)^{[1]},\left(x^{2 m+1}\right)^{[1]}\right), \\
H\left(\left(x^{i}\right)^{[1]}+\tau_{i}\left(x^{\bar{i}}\right)^{[1]},\left(x^{\bar{i}}\right)^{[1]},\left(x^{2 m+1}\right)^{[1]}\right)=H\left(\left(x^{i}\right)^{[1]},\left(x^{\bar{i}}\right)^{[1]},\left(x^{2 m+1}\right)^{[1]}\right) .
\end{gathered}
$$

Thus the maps $F_{k, \mu, \nu}$ and $H$ are independent of the first $m$ coordinates.

By the same argument for the $\left(\omega_{o}, \theta_{o}\right)$-automorphisms $\bar{x}^{\bar{i}}=x^{\bar{i}}-\tau_{i} x^{i}$, $\bar{x}^{i}=x^{i}$ and $\bar{x}^{2 m+1}=x^{2 m+1}$ for $i=1, \ldots, m$ we deduce that the maps $G_{k, \mu, \nu}$ and $H$ are independent of the next $m$ coordinates.

By the invariance of $\Lambda\left(\omega_{o}, \theta_{o}\right)$ with respect to the $\left(\omega_{o}, \theta_{o}\right)$-automorphisms $\bar{x}^{i}=\tau_{i} x^{i}, \bar{x}^{\bar{i}}=\left(1 / \tau_{i}\right) x^{\bar{i}}$ and $\bar{x}^{2 m+1}=x^{2 m+1}$ for $i=1, \ldots, m$ and arbitrary positive real numbers $\tau_{i}$ we get the homogeneity conditions

$$
\begin{aligned}
F_{k, \mu, \nu}\left(\tau_{i}\left(x^{\bar{i}}\right)^{[1]},\left(x^{2 m+1}\right)^{[1]}\right) & =\tau_{k} F_{k, \mu, \nu}\left(\left(x^{\bar{i}}\right)^{[1]},\left(x^{2 m+1}\right)^{[1]}\right), \\
G_{k, \mu, \nu}\left(\frac{1}{\tau_{i}}\left(x^{i}\right)^{[1]},\left(x^{2 m+1}\right)^{[1]}\right) & =\frac{1}{\tau_{k}} G_{k, \mu, \nu}\left(\left(x^{i}\right)^{[1]},\left(x^{2 m+1}\right)^{[1]}\right) .
\end{aligned}
$$

Now an application of the homogeneous function theorem completes the proof.

By (33) and Lemma 10 we can write

$$
\begin{aligned}
\Lambda\left(\omega_{o}, \theta_{o}\right) & \\
= & a\left(\left(x^{2 m+1}\right)^{[1]}\right)\left(\sum_{i=1}^{m} d\left(x^{i}\right)^{[0]} \wedge d\left(x^{\bar{i}}\right)^{[1]}-\sum_{i=1}^{m} d\left(x^{\bar{i}}\right)^{[0]} \wedge d\left(x^{i}\right)^{[1]}\right) \\
& +b\left(\left(x^{2 m+1}\right)^{[1]}\right) \sum_{i=1}^{m} d\left(x^{i}\right)^{[0]} \wedge d\left(x^{\bar{i}}\right)^{[0]} \\
& +\sum_{k=1}^{m} \sum_{\lambda, \mu=0,1} a_{k, \mu, \nu}\left(\left(x^{2 m+1}\right)^{[1]}\right)\left(x^{\bar{k}}\right)^{[1]} d\left(x^{k}\right)^{[\mu]} \wedge d\left(x^{2 m+1}\right)^{[\nu]}
\end{aligned}
$$




$$
\begin{aligned}
& +\sum_{k=1}^{m} \sum_{\lambda, \mu=0,1} b_{k, \mu, \nu}\left(\left(x^{2 m+1}\right)^{[1]}\right)\left(x^{k}\right)^{[1]} d\left(x^{\bar{k}}\right)^{[\mu]} \wedge d\left(x^{2 m+1}\right)^{[\nu]} \\
& +c\left(\left(x^{2 m+1}\right)^{[1]}\right) d\left(x^{2 m+1}\right)^{[0]} \wedge d\left(x^{2 m+1}\right)^{[1]}
\end{aligned}
$$

for some smooth maps $a, b, a_{k, \mu, \nu}, b_{k, \mu, \nu}, c: \mathbb{R} \rightarrow \mathbb{R}$.

LEMMA 11. We have

$$
a_{k, \mu, \nu}=-b_{k, \mu, \nu}=A_{\mu, \nu}
$$

for some smooth maps $A_{\mu, \nu}: \mathbb{R} \rightarrow \mathbb{R}$.

Proof. Using the invariance of $\Lambda\left(\omega_{o}, \theta_{o}\right)$ with respect to the $\left(\omega_{o}, \theta_{o}\right)$ automorphism $\bar{x}^{i}=-x^{\bar{i}}, \bar{x}^{\bar{i}}=x^{i}, \bar{x}^{2 m+1}=x^{2 m+1}$ for $i=1, \ldots, m$, from (37) we obtain $a_{k, \mu, \nu}=-b_{k, \mu, \nu}$. Next, using the invariance of $\Lambda\left(\omega_{o}, \theta_{o}\right)$ with respect to permutations of the first $m$ coordinates together with the corresponding permutations of the next $m$ coordinates on $\mathbb{R}^{2 m+1}$ we complete the proof.

Consider the $\left(\omega_{o}, \theta_{o}\right)$-diffeomorphism

$$
\psi=\left(x^{i}-\left(x^{\bar{i}}\right)^{2}, x^{\bar{i}}, x^{2 m+1}\right) .
$$

Lemma 12. For $i=1, \ldots, m$ we have

$$
\begin{aligned}
\left(x^{i}\right)^{[1]} \circ T \psi & =\left(x^{i}\right)^{[1]}, \\
\left(x^{\bar{i}}\right)^{[1]} \circ T \psi & =\left(x^{\bar{i}}\right)^{[1]}, \\
\left(x^{2 m+1}\right)^{[1]} \circ T \psi & =\left(x^{2 m+1}\right)^{[1]}, \\
d\left(x^{i}\right)^{[0]} \circ T T \psi & =d\left(x^{i}\right)^{[0]}, \\
d\left(x^{\bar{i}}\right)^{[0]} \circ T T \psi & =d\left(x^{\bar{i}}\right)^{[0]}, \\
d\left(x^{2 m+1}\right)^{[0]} \circ T T \psi & =d\left(x^{2 m+1}\right)^{[0]}, \\
d\left(x^{i}\right)^{[1]} \circ T T \psi & =d\left(x^{i}\right)^{[1]}-2\left(x^{\bar{i}}\right)^{[1]} d\left(x^{\bar{i}}\right)^{[0]}, \\
d\left(x^{\bar{i}}\right)^{[1]} \circ T T \psi & =d\left(x^{\bar{i}}\right)^{[1]}, \\
d\left(x^{2 m+1}\right)^{[1]} \circ T T \psi & =d\left(x^{2 m+1}\right)^{[1]}
\end{aligned}
$$

over $0 \in \mathbb{R}^{2 m}$.

The proof is standard and quite similar to the one of Lemma 7.

LEMMA 13. We have

$$
A_{1,0}=A_{1,1}=0 .
$$

Proof. This is a consequence of the invariance of $\Lambda\left(\omega_{o}, \theta_{o}\right)$ with respect to the $\left(\omega_{o}, \theta_{o}\right)$-diffeomorphism $\psi=\left(x^{i}-\left(x^{\bar{i}}\right)^{2}, x^{\bar{i}}, x^{2 m+1}\right)$. More precisely, we use $(37),(38)$ the invariance of $\Lambda\left(\omega_{o}, \theta_{o}\right)$ with respect to $\psi$ and the transformation rules (39)-(47). 
Proof of Theorem 4. By (48) and Lemmas 11 and 13 we have (4) with $(\omega, \theta)=\left(\omega_{o}, \theta_{o}\right)$ for some smooth maps $\alpha, \beta, \gamma, \delta, \varepsilon: \mathbb{R} \rightarrow \mathbb{R}$. Then an appeal to Lemma 8 completes the proof.

\section{References}

[1] J. Dębecki, Invariant vector fields of Hamiltonians, Arch. Math. (Brno) 34 (1988), 295-300.

[2] M. Doupovec, Natural liftings of (0,2)-tensor fields to the tangent bundle, ibid. 30 (1994),215-225.

[3] M. Doupovec and J. Kurek, Liftings of covariant (0,2)-tensor fields to the bundle of k-dimensional 1-velocities, Rend. Circ. Mat. Palermo (2) Suppl. 43 (1996), 111-121.

[4] M. Doupovec and J. Kurek, Natural operations of Hamiltonian type on the cotangent bundle, ibid. 46 (1997), 81-86.

[5] J. Grabowski and P. Urbański, Tangent lifts of Poisson and related structures, J. Phys. A 28 (1995), 6743-6777.

[6] I. Kolár, P. W. Michor and J. Slovák, Natural Operations in Differential Geometry, Springer, 1993.

[7] W. M. Mikulski, The geometrical constructions lifting tensor fields of type $(0,2)$ on manifolds to the bundles of A-velocities, Nagoya Math. J. 140 (1995), 117-137.

[8] W. M. Tulczyjew, Hamiltonian systems, Lagrangian systems, and the Legendre transformation, Symposia Math. 14 (1974), 101-114.

Institute of Mathematics

Maria Curie-Skłodowska University

Pl. Marii Curie-Skłodowskiej 1

20-031 Lublin, Poland

E-mail: kurek@golem.umcs.lublin.pl
Institute of Mathematics Jagiellonian University Reymonta 4 30-059 Kraków, Poland E-mail: mikulski@im.uj.edu.pl 